Feedback from patients and carers at our hospice-based MND support group highlighted that although the tertiary hospital clinic was important to them, it lacked access to local support, and pre-bereavement care. Often crises and issues arise in between hospital appointments; therefore local services are important in hospital admission avoidance where possible.

Aim To develop a satellite MND MDT clinic at our hospice incorporating the regional and local MND expertise.

Method The novel hospice-based clinic includes the hospital MND specialist nurse, an MND Association representative, and the hospice therapy team. New referrals are seen in the next monthly clinic.

The pilot will run for 12 months and be reviewed at six and 12 months.

During the year, clinic activity data, patient and carer feedback and a patient reported outcome scale (IPOS) will be collected as part of the service evaluation. This service is being set up within existing hospice budgets.

Results Preliminary analysis of IPOS and qualitative feedback data indicates that patients and carers prefer not having the burden of travelling to the tertiary hospital and value the opportunity to have more time to discuss their care and access the local services available to them. Frequent reviews and expedited access to hospice services has allowed more timely management of arising issues, preventing interval hospital admissions.

Conclusions By implementing the 2016 NICE Guidelines in MND care through our novel hospice-based clinic, we have a new service which rates very highly in satisfaction with patients and carers. It is also preventing crises in MND care falling upon tertiary centres by diversion to palliative care within local services.

\section{0-7 IMPROVING PRACTICE THROUGH AUDIT: ASSESSING AND MANAGING CONSTIPATION WITHIN A HOSPICE SETTING}

Joanne Rimmer, Aruna Hodgson, Jo Carby. Wigan and Leigh Hospice, Wigan, Greater Manchester

\subsection{6/bmjspcare-2016-001245.7}

Background Constipation is a common problem in palliative care, affecting approximately $50 \%$ of patients admitted to hospices and causing a wide range of symptoms. It is important to identify, treat and document constipation systematically in order to provide immediate symptomatic relief and to prevent complication occurrence.

Aims To identify if there is documented evidence that constipated hospice inpatients are being adequately assessed, monitored and managed, and to implement improvements where necessary.

Methods Retrospective audit of constipation assessment and management in twenty consecutive patients admitted to a hospice inpatient unit, against local and national standards. Data collected included documentation of bowel habits, oral laxative prescribing and administration, and rectal interventions.

Results A number of areas for improvement were identified in both medical and nursing documentation and clinical practice. Particular areas for improvement included standardisation of documentation, the creation and implementation of appropriate care plans, review and upward titration of oral laxatives, rectal medication administration, and obtaining verbal consent and offering a chaperone prior to bowel examination or intervention. Conclusions Lack of optimal assessment, management and documentation of constipation in hospice inpatients, will impact negatively on their physical and psychological symptom burden. In order to rectify this, a 'Constipation Working Party' was established comprising management, nursing and medical staff. Phase 1 of the improvement plan was the implementation of a 'Bowel Template' on the electronic patient record, on which all aspects of bowel care can be recorded. This has resulted in constipation documentation being more consistent, accurate and easily accessible. Phase 2 involves the development of hospice constipation guidelines, which are currently underway. Following completion and implementation of the new guidelines, a re-audit of constipation will be conducted to assess the extent to which the interventions have improved documentation and clinical practice.

\section{0-8 USABILITY AND ACCEPTABILITY OF AN ELECTRONIC PAIN MONITORING SYSTEM FOR ADVANCED CANCER: A THINK ALOUD STUDY}

Sally Taylor, Matthew Allsop, Bridgette Bewick, Michael Bennett. University of Leeds, Leeds, UK

\subsection{6/bmjspcare-2016-001245.8}

Introduction Pain experienced by advanced cancer patients is often poorly controlled due to inadequate monitoring or assessment. There is growing interest internationally in the development and implementation of remote monitoring technologies to enhance pain assessment. The aim of this study was to test an electronic pain monitoring system (PainCheck) with advanced cancer patients and health professionals and to explore how the system could be integrated into clinical practice.

Methods The think aloud method was used to gain an understanding of how patients and health professionals might use PainCheck. Twenty-nine participants (advanced cancer patients $(\mathrm{N}=13)$, GPs $(\mathrm{N}=4)$, community nurse specialists $(\mathrm{N}=4)$, district nurses $(\mathrm{N}=3)$, palliative care doctors $(\mathrm{N}=5))$ completed PainCheck while thinking their thoughts aloud. Patients completed the PainCheck assessment and explored their personalised feedback. Health professionals accessed a research version of PainCheck which contained reports from simulated patients. All participants were given minimal explanation of the system and were asked to use it as they would in their role as patient/ health professional. After the think aloud, both groups were asked about their experiences. Think-aloud and semi-structured interview data were analysed using framework analysis.

Results Health professionals were able to use PainCheck easily without instruction and were quickly able to consider its application into clinical practice. Patients needed a little more support when using PainCheck. Both groups identified potential benefits of using PainCheck such as improved monitoring and quicker access to advice and support. There were, however, some concerns about the potential burden and impact on time for patients and health professionals and fears that problems may be overlooked if PainCheck was not routinely monitored.

Conclusion PainCheck is acceptable to patients and health professionals but guidance on its use and the expectations of its users need to be clarified before it is integrated into clinical practice. 


\section{Conference Papers 3}

\section{0-9 REFLECTIONS ON AN EVOLVING @WEEOLC TWITTER COMMUNITY INFLUENCING CLINICAL PRACTICE, LEADERSHIP, QUALITY AND SELF}

${ }^{1}$ Sarah Russell, ${ }^{2}$ Bridget Johnston, ${ }^{3}$ Laura Green. ${ }^{1}$ Hospice UK, London, UK; ${ }^{2}$ School of Medicine, Dentistry and Nursing/University of Glasgow; ${ }^{3}$ Marie Curie/University of Bradford

\subsection{6/bmjspcare-2016-001245.9}

Background Three clinical academic nurses with palliative care backgrounds established @WeEOLC as part of the@WeNurses Twitter community in October 2015. The social media platform Twitter has the potential to influence care through the sharing of knowledge and practice as well as provoking discussion and debate (Russell et al., 2015). The focus of this abstract is to consider whether Twitter can assist in the achievement of improving evidence-based practice in palliative and end-of-life care through the learning outcomes framework of clinical practice, leadership, quality improvement and developing self (Taylor 2016).

Aim To share reflections on the value of engagements of @WeEOLC.

Method Established the twitter handle @WeEOLC \#WeEOLC to start and join in conversations. Scheduled regular chats with diverse guest hosts. Provided regular signposting of links to articles, blogs and resources. Analysis of chats and conversations. Results

\begin{tabular}{llll}
\hline Subject & Contributors & Number of Tweets & Reach \\
\hline The Big Conversation & 46 & 640 & $1,024,384$ \\
Archaeology & 32 & 476 & $1,399,106$ \\
Dementia & 63 & 626 & $1,346,221$ \\
Preferred Place of Death & 82 & 872 & $2,280,588$ \\
DNACPR: the family perspective & 76 & 865 & $2,258,274$ \\
Uncertainty & 85 & 808 & $4,750,670$ \\
\hline
\end{tabular}

Analysis of chats, contributor profiles and timelines provide rich insights into online activity. Simply counting the number of contributors and tweets does not reveal the full influence of @ WeEOLC. Chat and conversation contributors come from a wide background (e.g. clinicians, non-clinical, academics in social sciences, patients, families and interested parties). There is evidence of new connections and partnerships as well as a broad scope of interactive discussions, shared resources, evidence and reflections.

Conclusion Participation in platforms such as Twitter can support clinical and academic roles. By providing a non-hierarchical forum for learning and sharing, it contributes to clinical care, leadership and quality. Future work needs to develop methods of analysing and disseminating qualitative content as well as the quantitative reach of Twitter engagement.

\section{0-10 EFFECTIVENESS OF SOCIAL MEDIA VERSUS CLASSROOM-BASED EDUCATION IN PALLIATIVE AND END-OF-LIFE CARE TOPICS: MIXED METHODS STUDY}

\footnotetext{
${ }^{1}$ Munikumar Ramasamy Venkatasalu, ${ }^{2}$ Sarah Chapman, ${ }^{2}$ Hannah Dunn, ${ }^{2}$ Rachel Broad, ${ }^{3}$ Aruna Subramanim. ${ }^{1}$ Universiti Brunei Darussalam, Gadong, Brunei Darussalam; ${ }^{2}$ University of Bedfordshire, Aylesbury, UK; ${ }^{3}$ Sri Ramachandra University, Chennai, India
}

Background Online social networks act as a mode of communication that helps to share information and resources and collaborate with peers through engaging in social media based digital dialogues.

Aim To measure the effectiveness of social media and classroombased end-of-life care education among nursing students.

Methods A mixed methods approach was adopted. A total of 196 first-year undergraduate nursing students of one UK university were invited. 157 students joined Facebook based teaching and 34 students attended classroom-based end-of-life care teaching that delivered similar learning objectives. The Frommelt Attitude Toward Care of the Dying (FATCOD) Scale was completed by a group of nursing students before and after their participation in teaching sessions. Four post-teaching focus groups also conducted with students. Within the group, difference between preand post-test were assessed by using paired samples T-tests with use of SPSS V20. Using Nivio10 ${ }^{\circledR}$, a thematic qualitative analysis was undertaken.

Results Although there was no statistical significant difference in the level of attitude between the groups during pre-test, in posttest, there was a significant change in level of attitude for social media intervention group (94\% before vs 99\% after; $\mathrm{p}=0.041$ ) comparing to classroom-based education ( $84 \%$ before vs $81 \%$ after; $p=0.107)$. The mean difference score of Individual FATCOD items before and after education between two groups showed that the social media intervention group showed statistically significant change in student attitudes towards care of dying patients in 10 items. A comparative qualitative analysis revealed five themes: speaking out, lecture at living room, technology lessens interest, emotional teaching, and re-live lesson.

Conclusions Our study explored that using social media in death dying education enhances students' learning experiences both in emotional as well as knowledge acquisition similar to classroom teaching. However, Facebook teaching provided more flexibility and increased interest yet attained similar learning outcomes.

\section{0-11 MY LIFE: REPORTING ON A NOVEL USE OF DIGITAL LEGACY SOFTWARE IN HOSPICE DAY THERAPY}

${ }^{1}$ Nicola Denbow, ${ }^{2}$ Graham Stockton, ${ }^{1}$ Lisa Butterfield, 'Laura Green. 'Marie Curie, Bradford, UK; ${ }^{2}$ My Dementia Improvement Network

\subsection{6/bmjspcare-2016-001245.11}

Background Starting conversations about the end-of-life is known to be challenging for patients, families and professionals. The MyLife software contains a range of interactive therapeutic tools, including reminiscence abilities, social inclusion activities, and person centred, individualised "All About Me" passports. In addition, we are using the software to develop electronic life story memory books, that can be left as a legacy to loved ones. We are working with My Dementia Improvement Network to develop cloud-based storage to enable people's families from around the world to be able to contribute to the memory book. We are linking in with the local hospital dementia lead nurse to use the cloud to enable patients to continue to develop their books whilst in the hospice, hospital or at home.

Aim We saw an opportunity for this software to be useful in facilitating preparation for the end-of-life for patients and families using our hospice services.

Method Equipment was funded using a Yorkshire Young Achievers bid in 2014. We have trained four staff members as "superusers", by getting them to complete their own Life Story books. 